\title{
Thyroid hormone mediates cardioprotection against postinfarction remodeling and dysfunction through the IGF-1/PI3K/AKT signaling pathway
}

Bin Zeng ( $\square$ zengbinwhu0272008@163.com )

Wuhan University Renmin Hospital https://orcid.org/0000-0001-6847-5465

Xiaoting Liao

Renmin Hospital of Wuhan University

Lei Liu

1144698129@qq.com

Caixia Zhang

Renmin Hospital of Wuhan University

Huaiyu Ruan

Renmin Hospital of Wuhan University

\section{Research}

Keywords: Thyroid hormone, acute myocardial infarction, IGF-1/PI3K/AKT signaling, apoptosis, angiogenesis

Posted Date: April 29th, 2020

DOl: https://doi.org/10.21203/rs.3.rs-22987/v1

License: (c) (1) This work is licensed under a Creative Commons Attribution 4.0 International License.

Read Full License

Version of Record: A version of this preprint was published at Life Sciences on February $1 \mathrm{st}, 2021$. See the published version at https://doi.org/10.1016/j.lfs.2020.118977. 


\section{Abstract \\ Background}

Severe cardiovascular diseases, such as myocardial infarction or heart failure, can alter thyroid hormone (TH) secretion and peripheral conversion, leading to low triiodothyronine (T3) syndrome. Accumulating evidence suggests that $\mathrm{TH}$ has protective properties against cardiovascular diseases and that treatment with $\mathrm{TH}$ can effectively reduce myocardial damage after myocardial infarction (MI). However, the potential mechanisms are not clear. This study was designed to investigate the effect of T3 pretreatment on cardiac function and pathological changes in mice subjected to $\mathrm{Ml}$ and the underlying mechanisms.

\section{Methods}

Adult male C57BL/ 6 mice underwent surgical ligation of the left anterior descending coronary artery (LAD) (or sham operation) to establish a myocardial infarction model. T3, BMS-754807 (inhibitor of insulin-like growth factor-1 receptor (IGF-1R)) or vehicle was administered before surgery.

\section{Results}

Compared with the Ml group, the T3 pretreatment group exhibited significant attenuation of the myocardial infarct area, inhibition of cardiomyocyte apoptosis and fibrosis, and improved left ventricular function after MI. In addition, T3 exhibited an enhanced potency to stimulate angiogenesis and exert antiinflammatory effects by reducing the levels of serum inflammatory cytokines after myocardial infarction. However, all of these protective effects were inhibited by the IGF-1R inhibitor BMS-754807. Moreover, the protein expression of IGF-1/PI3K/AKT signaling-related proteins, such as IGF-1, IGF-1R, phosphorylated PI3K (p-PI3K) and p-AKT was significantly upregulated in MI mice that received T3 pretreatment, and BMS-754807 pretreatment blocked the upregulation of the expression of these signaling-related proteins.

\section{Conclusion}

T3 pretreatment can protect the heart against dysfunction post-MI through its anti-apoptotic, anti-fibrotic, anti-inflammatory and angiogenesis-stimulating effects, which may be mediated by the activation of the IGF-1/PI3K/AKT signaling pathway.

\section{Introduction}

Acute myocardial infarction (AMI) is a serious ischemic heart disease with significant morbidity and mortality worldwide[1]. Myocardial ischemia, cardiomyocyte apoptosis, fibrosis and aggravation of the inflammatory response are the main pathological changes contributing to ventricular remodeling and abnormalities in cardiac structure and function, which can seriously affect the quality of life and survival 
rate of patients[2]. In recent years, although a significant number of device-based interventions and chemical agents have been widely used in the clinic to ameliorate myocardial blood flow disturbance, the poor prognosis of $\mathrm{MI}$ remains a significant clinical problem[3]. Therefore, it is vital to develop new therapeutic strategies to reduce myocardial injury and aid cardiac repair following MI.

Thyroid hormone $(\mathrm{TH})$ is considered an important regulator of the cardiovascular system, which is essential for maintaining homeostasis of the heart and vascular system [4-6]. T3 is the most active component of $\mathrm{TH}$, and increasing evidence has indicated that $\mathrm{T} 3$ plays an important role in regulating and promoting cardiovascular function, including regulating cardiac hemodynamics, myocardial contractility, cardiac rhythm and vascular function[7-9]. In the clinical setting and experimental studies of acute myocardial infarction (AMI), the most common alteration in TH metabolism is a decrease in T3 levels. This occurs during the early stage of AMI and is associated with an increased incidence of adverse cardiac events[10]. Animal studies have confirmed that short-term moderate supplementation with T3 can reduce myocyte apoptosis and improve cardiac function after MI[5, 11], which indicates that T3 treatment could serve as a potential therapeutic approach for protecting against Ml. However, some studies have suggested that prolonged intake of high concentrations of T3 can lead to adverse factors such as myocardial hypertrophy and arrhythmia owing to the cardiotoxicity of $T 3[12,13]$. Thus, the optimal treatment duration of T3 is still worth exploring.

The thyroid hormone (TH)/insulin-like growth factor (IGF) signaling pathway has been identified in animals and humans. IGF-1 is a peptide hormone with structural similarity to insulin, which can regulate cardiac structure and function, such as inhibition of apoptosis, and promotion of cell proliferation [14]. Increasing evidence have suggested that T3 plays an important role in promoting IGF-1 binding to its receptor by regulating IGF-1 levels[15, 16]. When IGF-1 binds to IGF-1R, multiple phosphorylation cascades occur. As a consequence, $\mathrm{PI} 3 \mathrm{~K}$ is activated and AKT become phosphorylated. Phosphorylated AKT can attenuate apoptosis of cardiomyocytes and protect the myocardium from hypoxic ischemic injury $[14,17-19]$. Previous studies have shown that T3 can confer cardioprotection in mice post MI by activating the PI3K/AKT signaling pathway in cardiomyocytes $[5,20]$. However, whether the IGF1/P13K/AKT signaling pathway mediates the protective effect of T3 against cardiomyocyte damage caused by MI has not been reported. Therefore, the present study aimed to establish a model of myocardial infarction to study whether pretreatment with T3 can alleviate myocardial damage and promote cardiac repair after $\mathrm{Ml}$ and to explore the potential mechanisms behind it.

\section{Materials And Methods}

\section{Experimental Animals and Ethics Statement}

The animal protocols in this experiment were approved by the Animal Care and Use Committee of Renmin Hospital of Wuhan University and conformed to the Guidelines for the Care and Use of Laboratory Animals published by the US National Institutes of Health. Male C57BL/ 6 mice aged 8-10 weeks (weight: 24-29 g) were purchased from Beijing Vital River Laboratory Animal Technology Co., Ltd, (Beijing, China). 
Mice were housed with four to five mice in a cage fed with common food and water and maintained under $12 / 12 \mathrm{~h}$ light/dark cycles with the ambient humidity at $50-80 \%$ and the temperature at $21 \pm 2{ }^{\circ} \mathrm{C}$.

\section{Animal MI Model And Study Protocols}

The MI model was established by ligating the left anterior descending (LAD) coronary artery as described with minor modifications. Briefly, surgery was performed under anaesthesia with $3 \%$ sodium pentobarbital at a dose of $80 \mathrm{mg} / \mathrm{kg}$. A left thoracotomy was performed at the third or fourth intercostal space, open the pericardium, and the LAD was ligated with a $7-0$ silk suture at $2 \mathrm{~mm}$ below the end of left atrial appendage, decreased wall motion and paleness of Anterior wall of left ventricle below the ligation were recognized as a sign of successful ligation. For the sham operation, the left coronary artery was encircled but not ligated, after the surgery, the chest wall was closed, a heating pad was applied to maintain the core body temperature of the mouse at $36 \pm 1.0^{\circ} \mathrm{C}$. Lastly, the animals were put back in separate ventilated cages after they were fully recovered.

Male C57BL/6 mice aged 8-10 weeks were randomly divided into four groups:(1) Sham group (only received an injection of the same amount of saline); (2) MI group (only received an injection of the same amount of saline); (3) MI + T3 group (received an injection of T3(Sigma-Aldrich, USA) at the dose of $2 \mu \mathrm{g} / 100 \mathrm{~g} / \mathrm{d}$ ); (4) Ml + T3 + BMS-754807 group(received an injection of T3 at the dose of $2 \mu \mathrm{g} / 100 \mathrm{~g} / \mathrm{d}$ and BMS-754807 (Sellek Chemicals, USA) at the dose of $2 \mathrm{mg} / 100 \mathrm{~g} / \mathrm{d}$ ). Drugs or vehicle were administered intraperitoneally daily for 3 days before surgery, each mouse received the same total dose every day by adjusting the dose of saline. The number of animal deaths during the 7 days follow-up were carefully recorded.

\section{Echocardiographic Measurements}

Cardiac echocardiography was performed for the experimental mice at 7th day post operation. Mice in each group $(n=6)$ were anesthetised with $1.5-2 \%$ isoflurane by inhalation, and echocardiography was then performed using a MyLab30CV ultrasound (Biosound Esaote Inc. Indianapolis, IN, USA) with a 12.5$\mathrm{MHz}$ linear array ultrasound transducer. By adjusting the probe, a clear two-dimensional image of left ventricular short axis was obtained at the level of left ventricular papillary muscle. The left ventricular ejection fraction(LVEF), fractional shortening (FS), the left ventricular end-diastolic diameter (LVEDd), left ventricular end-systolic diameter(LVESd), left ventricular posterior wall thickness in diastole (LVPWd) and left ventricular posterior wall thickness in systole (LVPWs) were measured from M-mode recording . The parameters were calculated as mean of the measures obtained in at least three continuous cardiac cycles. All of the operators were blinded to the animal groups.

\section{Serum Inflammatory Cytokines Measurements}


Blood was collected from the left ventricle after function measurements, and then kept at room temperature for $1 \mathrm{~h}$, centrifuged at $2000 \mathrm{~g}$ for 10 minutes at $4{ }^{\circ} \mathrm{C}$, the buffy coat layer was collected and immediately stored at $-80^{\circ} \mathrm{C}$. Serum levels of inflammatory cytokines interleukin-6 (IL-6) and tumor necrosis factor alpha (TNF-a) were assayed by enzyme-linked immunosorbent assay(ELISA) kits(ELK Biotechnology, China)based on the manufacturer's instructions.

\section{Serum TH Measurements}

Serum levels of total T3 (TT3), free T3 (fT3), total T4 (TT4), free thyroxine (fT4) and thyrotropin (TSH) were assayed by ELISA kits based on the manufacturer's instructions.

\section{Tissue Harvest And Histological Analysis}

After function measurements were recorded, mice were euthanized with $3 \%$ sodium pentobarbital and the hearts were removed immediately, the excised hearts were placed in $10 \% \mathrm{KCl}$ solution to induce cardiac arrest during diastole, and then washed and excised the vessels and adipose tissue in ice-cold PBS, the weights of the heart and LV were recorded. Lungs were rapidly excised and weighed.

Masson's trichrome staining. To obtain cardiac pathological samples, the atriums above the ligation of the heart were removed, the remaining tissues were immersion-fixed with $4 \%$ paraformaldehyde and then processed by paraffin embedding. Subsequently, the hearts were cut serially from the base to the apex along the short axis. Each slice was cut at 5- $\mu$ m thickness. Sections were stained with Masson's trichrome stain (Solarbio Science and Technology, China) according to the manufacturer's protocol and then imaged. Morphometric analyses of the infarcted tissue were performed using Image-Pro Plus 6.0 software (Media Cybernetics, Inc. Rockville, MD, USA). Infarct size measurements were derived from the midpapillary level of the infarcted heart. Cardiac fibrosis measurements were obtained at the base, midpapillary, and apical regions of the heart. All measurements were made blindly.

\section{Immunofluorescence Staining}

TUNEL staining for myocardial apoptosis. For analyses of the activity of cardiomyocyte apoptosis, the paraffin sections were prepared, dried, dewaxed, hydrated and repaired, and then incubated with the following anti-bodies for confocal microscopy: Mouse Anti-Cardiac Troponin T antibody (Abcam, Britain; 1:250 dilution), Goat Anti-Mouse IgG H\&L (HRP) (Abcam, Britain; 1:250 dilution). Subsequently, the sections were washed in PBS, the TdT and dUTP in the TUNEL kit (Roche, Switzerland) were added to cover the tissue according to the instructions in the reagent kits, followed by DAPI (Invitrogen, USA) staining to label the nuclei. Lastly, sections were sealed with anti-fluorescence quenching sealant and placed under Olympus fluorescence microscope (Olympus Corporation, Tokyo, Japan) for image collection. whereas quantitative assessment and analysis were performed by counting the number of 
positive cells in the infarct border zone using Image-Pro Plus 6.0, the ratio of the number of apoptosispositive nuclei to total nuclei was used to represent the apoptosis rate.

Immunofluorescence of CD31. Sections obtained after preparation as described above were incubated with primary antibody of CD31(Abcam, Britain; 1:250 dilution) and the Cy3-conjugated secondary antibody (Abcam, Britain; 1:250 dilution) and DAPI (Invitrogen, USA) in sequence. Similarly, images were obtained by Olympus fluorescence microscope, the integrated density and area of CD31 of the infarct border zone were computed using Image-Pro Plus 6.0 software. The average optical densities (AODs) of CD31 were used to evaluate the density of angiogenesis and calculated as the integrated density/area multiplied by $100 \%$.

\section{Protein Extraction And Western Blot Analysis}

On the 7th day following surgery, the hearts were harvested and washed in PBS, the infarcted border zone of left ventricular were dissected and stored at $-80^{\circ} \mathrm{C}$. Cardiac tissues were minced, lysed with RIPA buffer(with protease and phosphatase inhibitors; Thermo Scientific, China), cracked on ice for 30 min and grinded with a homogenizer. The resulting homogenates were centrifugated at $13000 \mathrm{~g}$ for 5 minutes at $4{ }^{\circ} \mathrm{C}$ to collect protein supernatant. Protein concentrations were examined with bicinchoninic acid (BCA) protein assay kit (Rockford, USA). Protein extracts $(50 \mu \mathrm{g})$ were isolated by $12 \%$ SDS-PAGE (Invitrogen, USA) and then transferred to PVDF membrane (Millipore, USA). The membranes were sealed with $5 \%$ skimmed milk powder for $1 \mathrm{~h}$ at room temperature, and then incubated overnight at $4{ }^{\circ} \mathrm{C}$ with the primary antibodies(Cell Signaling Technology, Beverly, MA)specific to Bax (1:2000 dilution),Bcl-2 (1:500 dilution),Caspase-3 (1:1000 dilution),p-PI3K (1:500 dilution),p-AKT (1:1000 dilution). Subsequently, the membrane was washed with TBST for three times and incubated with a peroxidase-conjugated secondary antibody for $1 \mathrm{~h}$. Bands were visualized following activation with ECL (Thermo Scientific, USA), and the grey values of the bands represented the protein expression levels. The specific protein expression levels were normalised to $\beta$-actin on the same nitrocellulose membrane. Each group of experiments was repeated 3 times independently, and the average values were taken as the results.

\section{Statistical Analysis}

The experimental data were expressed by Mean + SEM and analyzed by SPSS 20.0 software (SPSS Inc., Chicago, IL, USA). Student unpaired $t$ test was used to compare two independent samples, and one-way analysis of variance (ANOVA) was used for comparison among multiple groups. If the overall difference was significant, post hoc Tukey's test was used to analyze the significant difference between the two groups. Values of $\mathrm{P}<0.05$ were considered statistically significant.

\section{Results}

\section{Changes of serum TH levels after T3 pretreatment}


Compared with Sham group, serum levels of tT3, fT3, tT4 and fT4 were significantly decreased after MI, and TSH increased $(P<0.05)$.T3 pretreatment increased the serum levels of total T3 and rT3, and significantly decreased the levels of tT4, fT4 and TSH compared with those of the MI group $(P<0.05)$, suggesting that T3 supplementation in vitro has negative feedback inhibition response on hypothalamicpituitary-thyroid axis. After the addition of BMS-754807, there was a tendency for decreased serum T3 levels as compared with the MI+T3 group (Fig. 1A-E).

\section{T3 pretreatment promotes survival rates and improves cardiac function after MI}

To specifically identify the effects of T3 on cardiac function induced by myocardial infarction, we established an MI model in mice and defined the endpoint as 7 days after the operation. In the sham group, none of the mice that underwent surgery died at the end of the observation period (100\% survival). However, cardiac rupture after Ml caused high mortality, and the survival rate through 7 days postoperation reached $68.5 \%$ in the $\mathrm{Ml}$ group $(\mathrm{P}<0.05)$. T3 pretreatment increased the survival rate after MI (80.2\%; $\mathrm{P}<0.05)$, but BMS-754807 suppressed this effect; the MI + T3 + BMS-754807 group had the lowest survival rate of $62.5 \%(P<0.05$; Fig. $2 A)$.

To assess global systolic and diastolic function, we performed echocardiography studies 7 days after the operation. The results indicated that left ventricular function was impaired after MI. Compared with MI animals, T3 pretreatment animals exhibited preserved LVEF and FS and reduced LVESd and LVEDd values $(\mathrm{P}<0.05)$. In contrast, BMS-754807 suppressed the effects of T3 stimuli and aggravated MIinduced cardiac dysfunction $(P<0.05)$. Furthermore, LVPWs and LVPWd, which were assessed by echocardiography, were obviously reduced in MI mice compared to sham mice, but T3 pretreatment significantly reduced the changes in LV wall thickness during systole and diastole $(P<0.05)$, suggesting that T3 pretreatment can bring sustained functional and structural benefits. There was no significant difference in heart rate between each group before operation. Heart rate obviously increased after operation compared with the sham animals, but there was no significant difference in the last three groups ( $P>0.05$; Fig. 3A-F).

\section{T3 pretreatment reduces infarct size and ameliorates myocardial fibrosis after MI}

Masson's trichrome staining at the was performed at the endpoint to evaluate infarct size, myocardial fibrosis and structural changes. The results showed that infarct size and fibrosis were dramatically increased in the $\mathrm{MI}$ group $(31.3 \%$ and $29.8 \%)$ compared to the sham group $(0 \%)(P<0.05)$ and that T3 infusion markedly reduced infarct size and limited myocardial fibrosis spaces and collagen volumes after MI $(22.6 \%$ and $21.9 \% ; \mathrm{P}<0.05)$. Conversely, BMS-754807 suppressed this effect, resulting in a larger infarct area and more severe myocardial fibrosis in the MI + T3 + BMS-754807 group ( $37.4 \%$ and $36.7 \%$ ) $(P<0.05$; Fig. 4A and $B)$.

\section{T3 Pretreatment Attenuates Ml-induced Myocardial Apoptosis}


The extent of apoptosis was detected through a TUNEL assay in peri-infarct tissues. Notably, TUNELpositive nuclei were rarely observed in the sham group. However, the number of TUNEL-positive nuclei was significantly increased after MI. The ratio of TUNEL-positive nuclei was $28.6 \%$ in the $\mathrm{MI}$ group, and T3 pretreatment greatly reduced the ratio of TUNEL-positive nuclei to $14.1 \%(P<0.05)$, suggesting that T3 protects cardiomyocytes against Ml-induced apoptosis. However, BMS-754807 inhibited the effect of T3, and the ratio of TUNEL-positive cells in the $\mathrm{Ml}+\mathrm{T} 3+\mathrm{BMS}-754807$ group was $35.9 \%(\mathrm{P}<0.05$, Fig. $5 \mathrm{~A}$ and $B)$. We also examined the expression levels of apoptosis-related proteins, including pro-apoptotic factors (Bax and cleaved caspase 3) and anti-apoptotic factors (Bcl-2), to clarify the anti-apoptotic effect of T3. Western blot analyses revealed that the expression of Bax and cleaved caspase- 3 was significantly increased, while the expression of $\mathrm{Bcl}-2$ was decreased in the $\mathrm{Ml}$ group compared with the sham group. Conversely, T3 infusion markedly suppressed Bax and cleaved caspase 3 expression and preserved Bcl-2 expression compared with that in the $\mathrm{Ml}$ group, and relative to animals treated with saline or $\mathrm{T} 3$ infusion, the $\mathrm{Ml}+\mathrm{T} 3+\mathrm{BMS}-754807$ group showed higher expression of Bax and cleaved caspase 3 and the lower expression of Bcl-2 (all $\mathrm{P}<0.05$, Fig. $5 \mathrm{C}$ and $\mathrm{D}$ ).

\section{T3 Pretreatment Inhibits Ml-induced Inflammatory Responses}

We examined the serum levels of inflammatory cytokines (IL-6 and TNF-a) to explore whether T3 can affect the inflammatory response in the heart after MI. In this study, the levels of inflammatory cytokines were much higher in mice subjected to $\mathrm{Ml}$ than in mice that underwent sham operation, indicating that the degree of inflammatory cytokine release was greatly enhanced after $\mathrm{MI}(\mathrm{P}<0.05)$. Interestingly, the mice in the $\mathrm{Ml}+\mathrm{T} 3$ group exhibited obviously decreased levels of inflammatory markers in comparison with those exhibited by the mice the Ml group $(P<0.05)$. In contrast, the levels of inflammatory cytokines were clearly increased in the MI + T3 + BMS-754807 group compared to the $\mathrm{Ml}+\mathrm{T} 3$ group $(\mathrm{P}<0.05$, Fig. $6 \mathrm{~A}$ and $\mathrm{B})$.

\section{T3 Pretreatment Stimulates Angiogenesis Following MI}

The number of cells positively stained for CD31 represented the density of new blood vessels. Compared with the sham group, the AODs of CD31 in the MI group were significantly decreased ( $66.4 \%$ VS $42.3 \%$; $P$ $<0.05)$. T3 pretreatment markedly increased the AODs of CD31 to $58.1 \%$ compared with those in the MI group $(P<0.05)$, but BMS-754807 significantly diminished the effect of T3. The AODs of CD31 in the MI+ T3 + BMS-754807 group were the lowest in MI mice (40.9\%; P<0.05, Fig. 7A and B).

T3 pretreatment mediates post-MI cardioprotection via the activation of the IGF-1/PI3K/AKT signaling pathway

Growing evidence has demonstrated that the IGF-1/PI3K/AKT signaling pathway plays an important role in regulating cardiovascular diseases. Therefore, signaling pathway-related proteins were detected to 
investigate the possible association between the IGF-1/PI3K/AKT signaling pathway and the cardioprotective effects of T3. In the current study, there was no significant difference in the protein expression of total PI3K or total AKT between the four groups, but the protein expression levels of IGF-1, IGF-1R, p-PI3K and p-AKT were increased in the model groups in comparison with those in the sham group. Notably, pretreatment with T3 significantly upregulated the expression levels of the signalingrelated proteins mentioned above compared with those in the Ml group, suggesting that pretreatment with T3 may activate the IGF-1/PI3K/AKT signaling pathway. BMS-754807 pretreatment blocked this effect mediated by $\mathrm{T} 3$, and the expression of relative proteins was greatly decreased in the $\mathrm{MI}+\mathrm{T} 3+\mathrm{BMS}-$ 754807 group (all $\mathrm{P}<0.05$; Fig. 8 A and $B$ ).

\section{Discussion}

The present study shows that T3 pretreatment confers cardioprotection on mice subjected to MI. These protective effects are associated with improved cardiac function, decreased cardiomyocyte apoptosis and infarct size, limited LV remodeling, stimulated angiogenesis, and a blunted inflammatory response. Pretreatment with T3 can activate the IGF-1/PI3K/AKT signaling pathway, which is partially responsible for these protective effects. Therefore, T3 pretreatment might represent a promising therapeutic approach for MI.

The results of this study showed that the serum levels of tT3, fT3, tT4 and fT4 were significantly decreased and TSH increased after MI, which was consistent with previous studies[5, 7]. T3 pretreatment increased the serum levels of total T3 and rT3, and significantly decreased the levels of tT4, fT4 and TSH compared with those of the Ml group, suggesting that T3 supplementation in vitro has negative feedback inhibition response on hypothalamic-pituitary-thyroid axis.

Thyroid hormones have long been known to have a range of beneficial effects on the cardiovascular system[6, 10,20,21].Some evidence has emerged in support of the concept that the elevation of cardiac TH levels can enhance myocardial contractility and ameliorate the impairment of cardiac function caused by MII22, 23]. The present study confirmed that T3 pretreatment dramatically improved the survival rates of mice subjected to MI. As expected, echocardiographic evaluations showed obviously elevated LVEF and FS values and reduced LVESd values in T3 pretreatment mice, indicating that T3 pretreatment significantly improved left ventricular function in mice after MI. However, there was no significant difference in heart rate between the two groups, suggesting that the T3 pretreatment-induced increase in the values of LVEF in MI animals may not be associated with heart rate. Thus, the increase in myocardial contractility induced by T3 may be explained at the genetic level, for example, by the stimulation of aMHC expression and the repression of $\beta \mathrm{MHC}$ expression to increase intracellular $\mathrm{Ca} 2+$ concentration to enhance contractility $[4,24]$.

Masson's trichrome staining was used to directly assess the degree of structural injury in mice. Compared with the MI group, the T3 pretreatment group exhibited marked reductions in the infarct size and changes in the LV wall thickness during systole and diastole after MI, suggesting that T3 pretreatment can induce 
sustained structural benefits. Ischemia and hypoxia can affect myocardial collagen metabolism and scar contraction. In response to MI injury, LV remodeling can increase diastolic stiffness and impair relaxation, which further aggravates cardiac dysfunction [25-27]. Our study showed that myocardial interstitial fibrosis increased after MI but that T3 pretreatment significantly reduced myocardial fibrosis space and collagen volume after $\mathrm{Ml}$, which suggested that $\mathrm{T} 3$ has an anti-remodeling effect and that this effect might contribute to the improvement of LV relaxation.

Accumulating evidence has demonstrated that a sudden increase in cardiomyocyte apoptosis occurs in the ischemic hearts of both patients and animals and that apoptosis leads to continuous cardiomyocyte loss throughout the entire pathological process post-MI[28]. Therefore, the inhibition of cardiomyocyte apoptosis has shown to be potentially protective against post-MI cardiac dysfunction. In this study, the ratio of TUNEL-positive nuclei was significantly decreased in T3-pretreated mice, indicating that T3 infusion significantly reduced myocardial apoptosis. Moreover, T3 infusion markedly suppressed the expression of the pro-apoptotic factors Bax and cleaved caspase 3 and preserved the expression of the anti-apoptotic gene Bcl-2 compared to that in the MI group, which further confirmed the anti-apoptotic effects of $\mathrm{T} 3$ in cardiomyocytes during Ml insult.

It has been well documented that innate immune and inflammatory responses are involved in the pathological processes of $\mathrm{MI}$ and ultimately lead to healing and scar formation [25, 29, 30]. Studies have shown that thyroid hormones can modulate the inflammatory response through genomic and nongenomic mechanisms and that low T3 circulating levels usually indicate more severe inflammatory responses in patients with MI[31,32]. We focused on the traditional inflammatory cytokine interleukin 6 (IL-6) and tumor necrosis factor-a (TNF-a), elevated levels of which are considered to be associated with a more severe clinical status and a worse outcome. The results showed that these plasma cytokines were distinctly elevated after MI but that T3 infusion significantly reduced the levels of these inflammatory cytokines and played an anti-inflammatory role. Considering that inflammatory infiltration into the noninfarcted myocardium may worsen fibrosis and that apoptosis of cardiomyocytes is accompanied by the induction of interstitial fibrosis, reductions in inflammatory and apoptotic responses might be responsible for the preventive effect of T3 on LV remodeling and subsequent cardiac dysfunction[29, 30].

Angiogenesis is a complex process in which new capillary blood vessels and small non-muscular blood vessels grow through the proliferation and migration of endodermal vesicles[33]. The formation of capillary network in the infarcted border zone can improve the blood supply to the ischemic myocardium[34, 35]. Changes in local metabolic status, such as hypoxia, also promote angiogenesis [36]. CD31 is a specific angiogenesis marker expressed in endothelial cells and can reflect microvascular density[35]. Our results demonstrated that T3 pretreatment significantly increased the AODs of CD31 in the infarcted border zone in mice post-MI compared with MI mice, which suggested that T3 can markedly promote the proliferation of collateral vessels in ischemic areas and improve blood circulation to reduce infarct size and promote the repair of cardiac injury. 
It is believed that the IGF-1/PI3K/AKT signaling pathway is a critical signaling cascade that promotes cell survival $[14,20]$. A number of studies have discovered that supplementation with TH can lead to activation of the IGF-1/PI3K/AKT signaling pathway by promoting the binding of IGF-1 to IGF-1R and then effectively inhibit cardiomyocyte apoptosis, attenuate myocardial remodeling, reduce inflammation and initiate angiogenesis after cardiac ischemia injury $[17,18]$. In the present study, compared with those in the MI group, the protein expression levels of IGF-1, IGF-1R, p-PI3K and p-AKT were significantly upregulated after T3 pretreatment, indicating that T3 activated the IGF-1/PI3K/AKT signaling pathway and then regulated the signaling pathway to protect cardiomyocytes.

BMS-754807, a potent inhibitor of IGF-1R, eliminated all of the protective effects of T3 mentioned above. The MI + T3 + BMS-754807 group had the lowest survival rate, the worst cardiac function, and the worst structural and pathological changes. Meanwhile, the expression levels of IGF-1, IGF-1R, p-AKT and p-PI3K were significantly reduced compared with those in the MI + T3 group, indicating that BMS-754807 counteracted the phosphorylation of PI3K/AKT mediated by T3. Thus, the conclusion that the protective effect of T3 in reducing MI-induced cardiomyocyte injury is mediated by the activation of the IGF1/PI3K/AKT signaling pathway is clear.

\section{Conclusions}

In conclusion, this study presents the first evidence that T3 pretreatment plays a role in attenuating the myocardial infarct area, inhibiting cardiomyocyte apoptosis and fibrosis, stimulating angiogenesis and protecting against cardiac dysfunction after myocardial infarction. The underlying mechanism of the protective role of T3 in the development of myocardial injury post-MI involves the activation of the IGF1/PI3K/AKT signaling pathway. Therefore, we propose that T3 pretreatment is a promising approach for treating postinfarction cardiac dysfunction and cardiac remodeling.

\section{Abbreviations}

TH thyroid hormone

T3 triiodothyronine

MI myocardial infarction

AMI acute myocardial infarction

LAD left anterior descending

IGF-1 insulin-like growth factor-1

IGF-1R insulin-like growth factor-1 receptor

PI3K phosphatidylinositol 3-hydroxy kinase 
p-PI3K phosphorylated-phosphatidylinositol 3-hydroxy kinase

AKT protein kinase $B$

p-AKT phosphorylated-protein kinase B

HW heart weight

LW lung weight

BW body weight.

LVEF left ventricular ejection fraction

FS fractional shortening

LVEDd the left ventricular end-diastolic diameter

LVESd left ventricular end-systolic diameter

LVPWd left ventricular posterior wall thickness in diastole

LVPWs left ventricular posterior wall thickness in systole

HR Heart rate

IL-6 interleukin-6

TNF-a tumor necrosis factor-alpha

AODs average optical densities

\section{Declarations}

\section{Ethics approval and consent to participate}

The animal protocols in this experiment were approved by the Animal Care and Use Committee of Renmin Hospital of Wuhan University and conformed to the Guidelines for the Care and Use of Laboratory Animals published by the US National Institutes of Health. License no. SCXKX02015-0027.

\section{Consent for publication}

All the authors confirm that the work described has not been published before, and all the authors agree to publication in the Journal .

\section{Availability of data and material}


Male C57BL/6 mice were purchased from Beijing Vital River Laboratory Animal Technology Co., Ltd. (Certificate of quality N0.11400700323271).Datas and materials are valid.

\section{Author contributing}

Bin Zeng and Xiaoting Liao designed the experiment, Xiaoting Liao carried out the experiments, Lei Liu, Caixia Zhang and Huaiyu Ruan conceived and coordinated the study, Xiaoting Liao wrote the manuscript. All authors reviewed the results and approved the final version.

\section{Acknowledgements}

This work was supported by Department of Cardiology, Renmin Hospital of Wuhan University, Cardiovascular Research Institute, Wuhan University, Hubei Key Laboratory of Cardiology.

\section{Funding}

This work was supported by the Chinese National Nature Science Foundation $(30900609,81270271$ and 81570333).

\section{Disclosure}

None.

\section{Conflict of interest}

No conflicts of interest were declared.

\section{References}

1. Heusch G. Cardioprotection: chances and challenges of its translation to the clinic. Lancet. 2013;381(9861):166-75.

2. Li L, et al., Regulatory role of CARD3 in left ventricular remodelling and dysfunction after myocardial infarction. Basic Research in Cardiology, 2015. 110(6).

3. Hausenloy DJ, et al. Cardiac innervation in acute myocardial ischaemia/reperfusion injury and cardioprotection. Cardiovasc Res. 2019;115(7):1167-77.

4. Razvi S, et al. Thyroid Hormones and Cardiovascular Function and Diseases. J Am Coll Cardiol. 2018;71(16):1781-96.

5. Chen Y, et al. Short term triiodo-l-thyronine treatment inhibits cardiac myocyte apoptosis in border area after myocardial infarction in rats. J Mol Cell Cardiol. 2008;44(1):180-7.

6. Cappola AR, et al. Thyroid and Cardiovascular Disease: Research Agenda for Enhancing Knowledge, Prevention, and Treatment. Thyroid. 2019;29(6):760-77. 


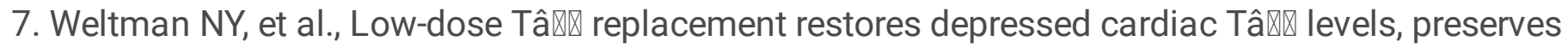
coronary microvasculature and attenuates cardiac dysfunction in experimental diabetes mellitus. Molecular medicine (Cambridge, Mass.), 2014. 20(1): p. 302-312.

8. Pingitore A, et al. Cardioprotection and thyroid hormones. Heart Fail Rev. 2016;21(4):391-9.

9. Rajagopalan V, et al., Modified Low-Dose Triiodo-L-thyronine Therapy Safely Improves Function Following Myocardial Ischemia-Reperfusion Injury. Frontiers in Physiology, 2017. 8.

10. Pingitore A, lervasi G, Forini F. Role of the Thyroid System in the Dynamic Complex Network of Cardioprotection. European cardiology. 2016;11(1):36-42.

11. Chen YF, et al. Improvement of left ventricular remodeling after myocardial infarction with eight weeks L-thyroxine treatment in rats. J Transl Med. 2013;11:40.

12. Seara FAC, et al. Cardiac ischemia/reperfusion injury is inversely affected by thyroid hormones excess or deficiency in male Wistar rats. PLOS ONE. 2018;13(1):e0190355.

13. Kuzman JA, Connell TDO, Gerdes AM. Rapamycin Prevents Thyroid Hormone-Induced Cardiac Hypertrophy. Endocrinology. 2007;148(7):3477-84.

14. Ge P, et al. L-carnitine affects osteoblast differentiation in NIH3T3 fibroblasts by the IGF-1/PI3K/Akt signalling pathway. BioScience Trends. 2015;9(1):42-8.

15. Xing W, et al. Genetic evidence that thyroid hormone is indispensable for prepubertal insulin-like growth factor-I expression and bone acquisition in mice. J Bone Miner Res. 2012;27(5):1067-79.

16. Wang $Y$, Zhang S. Expression and regulation by thyroid hormone (TH) of zebrafish IGF-I gene and amphioxus IGFI gene with implication of the origin of TH/IGF signaling pathway. Comp Biochem Physiol A: Mol Integr Physiol. 2011;160(4):474-9.

17. $\mathrm{He} \mathrm{H}$, et al. Salidroside Mitigates Sepsis-Induced Myocarditis in Rats by Regulating IGF1/PI3K/Akt/GSK-3ß Signaling. Inflammation. 2015;38(6):2178-84.

18. Wen Z, et al. Angiotensin II receptor blocker reverses heart failure by attenuating local oxidative stress and preserving resident stem cells in rats with myocardial infarction. American journal of translational research. 2018;10(8):2387-401.

19. Cantley LC. The phosphoinositide 3-kinase pathway. Science. 2002;296(5573):1655-7.

20. Pantos C, Mourouzis I, Cokkinos DV. New insights into the role of thyroid hormone in cardiac remodeling: time to reconsider? Heart Fail Rev. 2011;16(1):79-96.

21. Pantos $C$, et al. Thyroid hormone improves postischaemic recovery of function while limiting apoptosis: a new therapeutic approach to support hemodynamics in the setting of ischaemiareperfusion? Basic Res Cardiol. 2009;104(1):69-77.

22. Zhang K, et al. Comparison of Therapeutic Triiodothyronine Versus Metoprolol in the Treatment of Myocardial Infarction in Rats. Thyroid. 2018;28(6):799-810.

23. Trivieri MG, et al. Cardiac-Specific Elevations in Thyroid Hormone Enhance Contractility and Prevent Pressure Overload-Induced Cardiac Dysfunction. Proc Natl Acad Sci USA. 2006;103(15):6043-8. 
24. Trivieri MG, et al. Cardiac-specific elevations in thyroid hormone enhance contractility and prevent pressure overload-induced cardiac dysfunction. Proc Natl Acad Sci U S A. 2006;103(15):6043-8.

25. Sutton MG, Sharpe N. Left ventricular remodeling after myocardial infarction: pathophysiology and therapy. Circulation. 2000;101(25):2981-8.

26. van den Borne SW, et al. Myocardial remodeling after infarction: the role of myofibroblasts. Nat Rev Cardiol. 2010;7(1):30-7.

27. $\mathrm{Li}$ L, et al., Regulatory role of CARD3 in left ventricular remodelling and dysfunction after myocardial infarction. Basic Research in Cardiology, 2015. 110(6).

28. Dorn GW. Apoptotic and non-apoptotic programmed cardiomyocyte death in ventricular remodelling. Cardiovasc Res. 2008;81(3):465-73.

29. Pantos C, Mourouzis I, Cokkinos DV. Thyroid hormone as a therapeutic option for treating ischaemic heart disease: From early reperfusion to late remodelling. Vascul Pharmacol. 2010;52(3-4):157-65.

30. López $\mathrm{E}$, et al., Identification of very early inflammatory markers in a porcine myocardial infarction model. BMC Veterinary Research, 2019. 15(1).

31. De Vito P, et al. Thyroid Hormones as Modulators of Immune Activities at the Cellular Level. Thyroid. 2011;21(8):879-90.

32. Lubrano V, et al. Relationship between triiodothyronine and proinflammatory cytokines in chronic heart failure. Biomed Pharmacother. 2010;64(3):165-9.

33. Kimura T, et al. Involvement of circulating interleukin- 6 and its receptor in the development of euthyroid sick syndrome in patients with acute myocardial infarction. European journal of endocrinology. 2000;143(2):179-84.

34. Badimon L, Borrell M. Microvasculature Recovery by Angiogenesis After Myocardial Infarction. Curr Pharm Des. 2018;24(25):2967-73.

35. Sass FA, et al. CD31 + Cells From Peripheral Blood Facilitate Bone Regeneration in Biologically Impaired Conditions Through Combined Effects on Immunomodulation and Angiogenesis. J Bone Miner Res. 2017;32(5):902-12.

36. Cochain C, Channon KM, Silvestre J. Angiogenesis in the Infarcted Myocardium. Antioxid Redox Signal. 2013;18(9):1100-13.

\section{Figures}




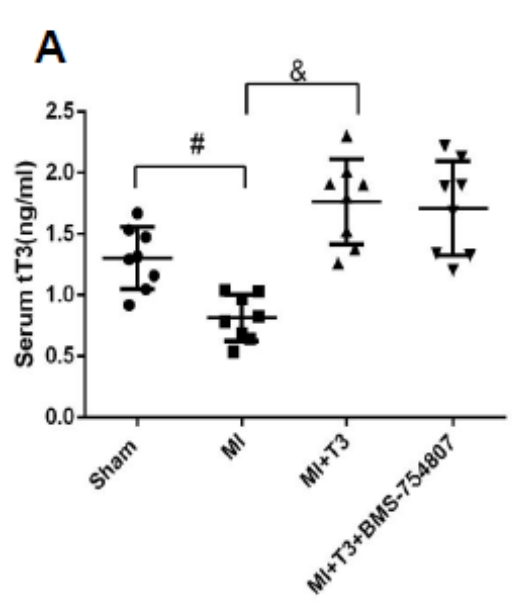

B

C

D
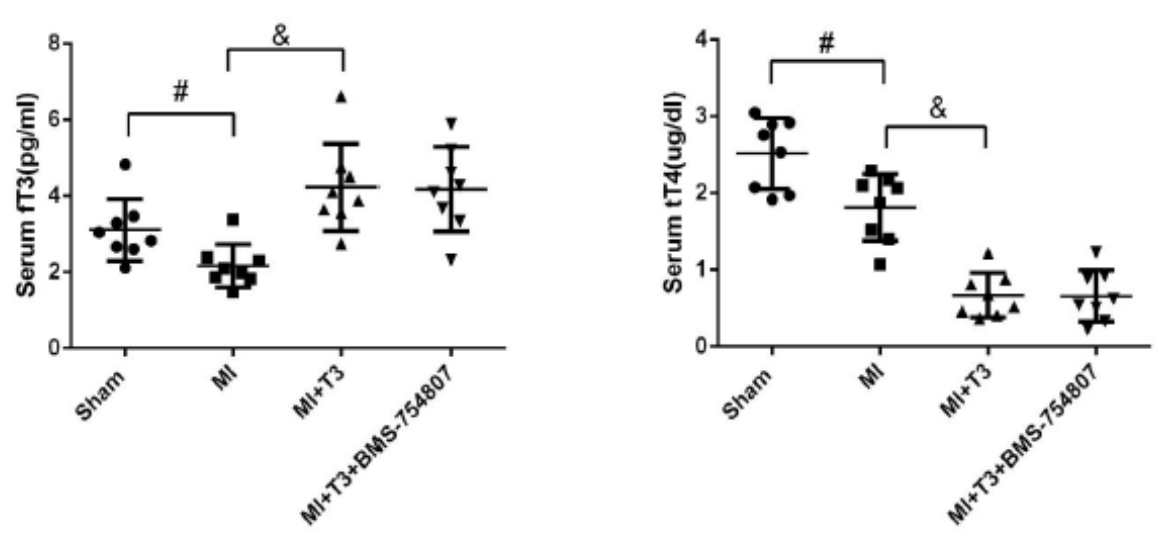

$\mathbf{E}$
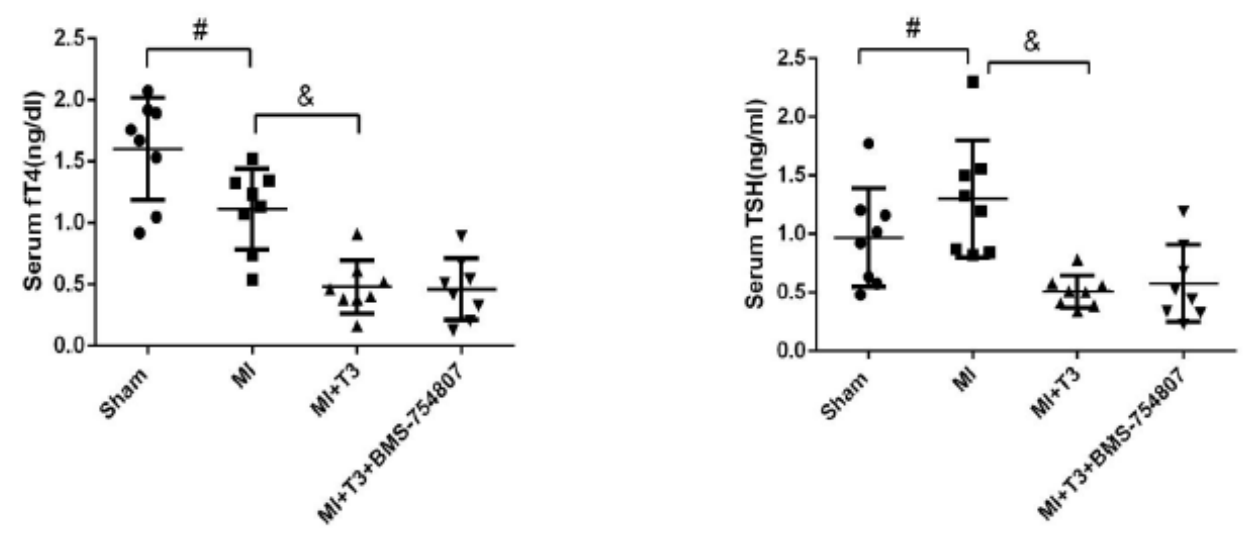

Figure 1

Serum TH levels. (A) total triiodothyronine (TT3); (B) free triiodothyronine (fT3); (C) total thyroxine (TT4); (D) free thyroxine (fT4); (E) thyrotropin (TSH). ( $=8$ per group). \# $P<0.05$ versus sham group; \& $P<0.05$ versus $\mathrm{Ml}$ group, * $\mathrm{P}<0.05$ versus $\mathrm{Ml}+\mathrm{T} 3$ group. 


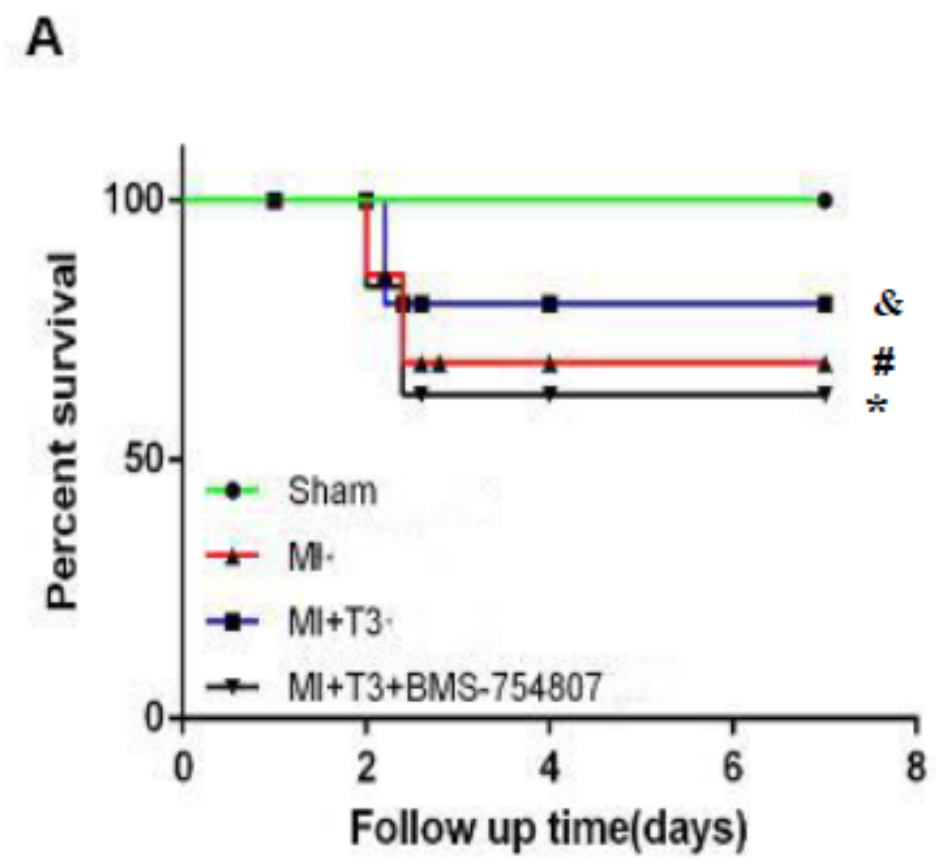

Figure 2

T3 pretreatment promotes survival rates after MI. (A) Kaplan-Meier survival analysis of mice through 7 days after operation. \# $\mathrm{P}<0.05$ versus sham group; $\& \mathrm{P}<0.05$ versus $\mathrm{MI}$ group, * $\mathrm{P}<0.05$ versus $\mathrm{Ml}+\mathrm{T} 3$ group. BMS, BMS-754807. 
A

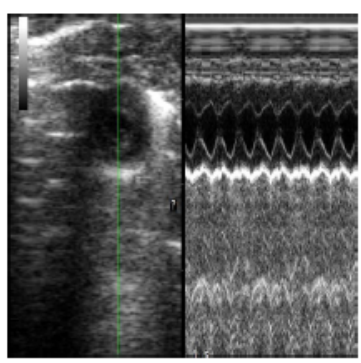

c

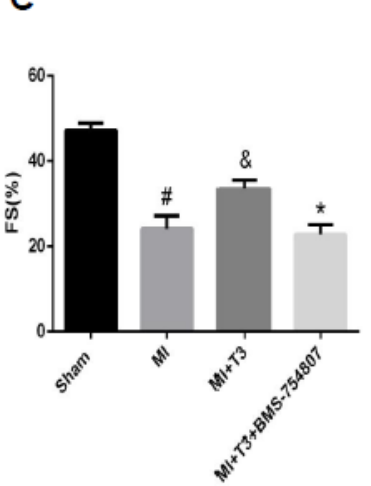

MI

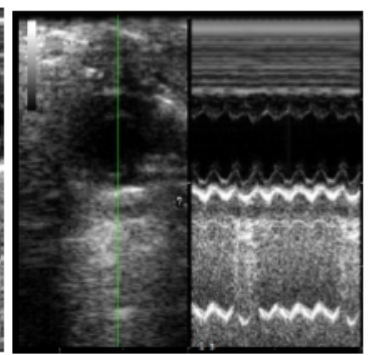

D

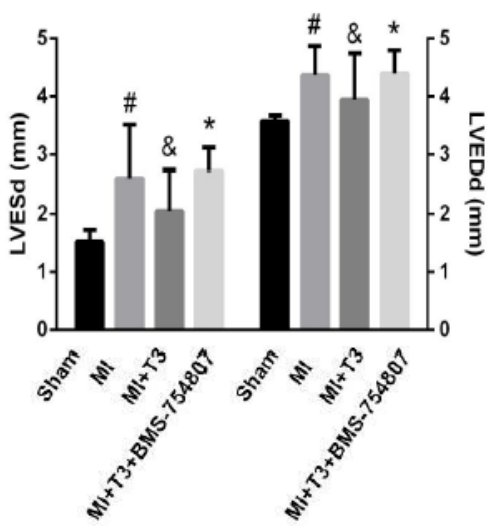

$\mathrm{Ml}+\mathrm{T3}$

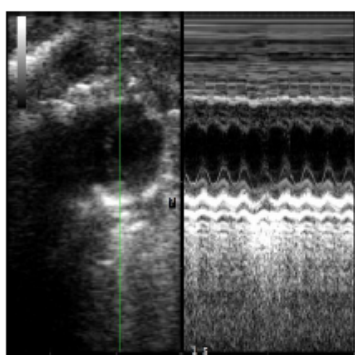

E

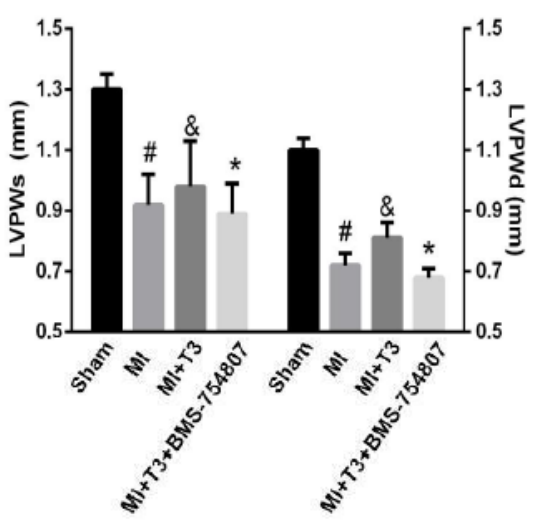

B

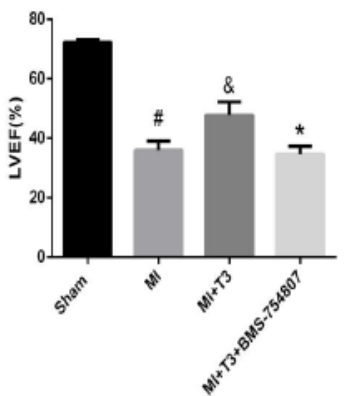

F

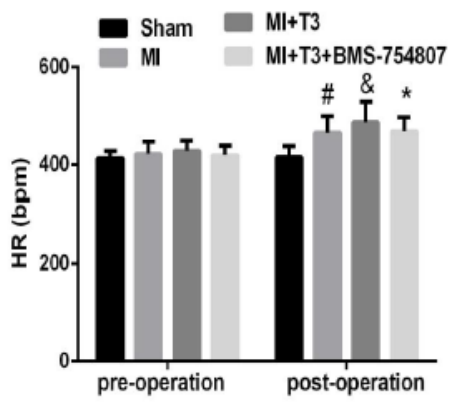

\section{Figure 3}

T3 pretreatment improves cardiac function after MI. (A) Left ventricular mode echocardiograms in short axis at 7th day after operation. (B) Left ventricular ejection fraction (\%LVEF). (C) Fractional shortening (\%FS). (D) Left ventricular end-systolic diameter (LVESd). (E) Left ventricular end-diastolic diameter (LVEDd). (F) Left ventricular posterior wall thickness in systole (LVPWs). (G) Left ventricular posterior wall thickness in diastole (LVPWd). (H) Heart rate (HR). Data are expressed as mean \pm SEM ( $\mathrm{n}=8$ per group). \# $\mathrm{P}<0.05$ versus sham group; \& $\mathrm{P}<0.05$ versus $\mathrm{Ml}$ group, * $\mathrm{P}<0.05$ versus $\mathrm{Ml}+\mathrm{T} 3$ group. 
$(200 X)$
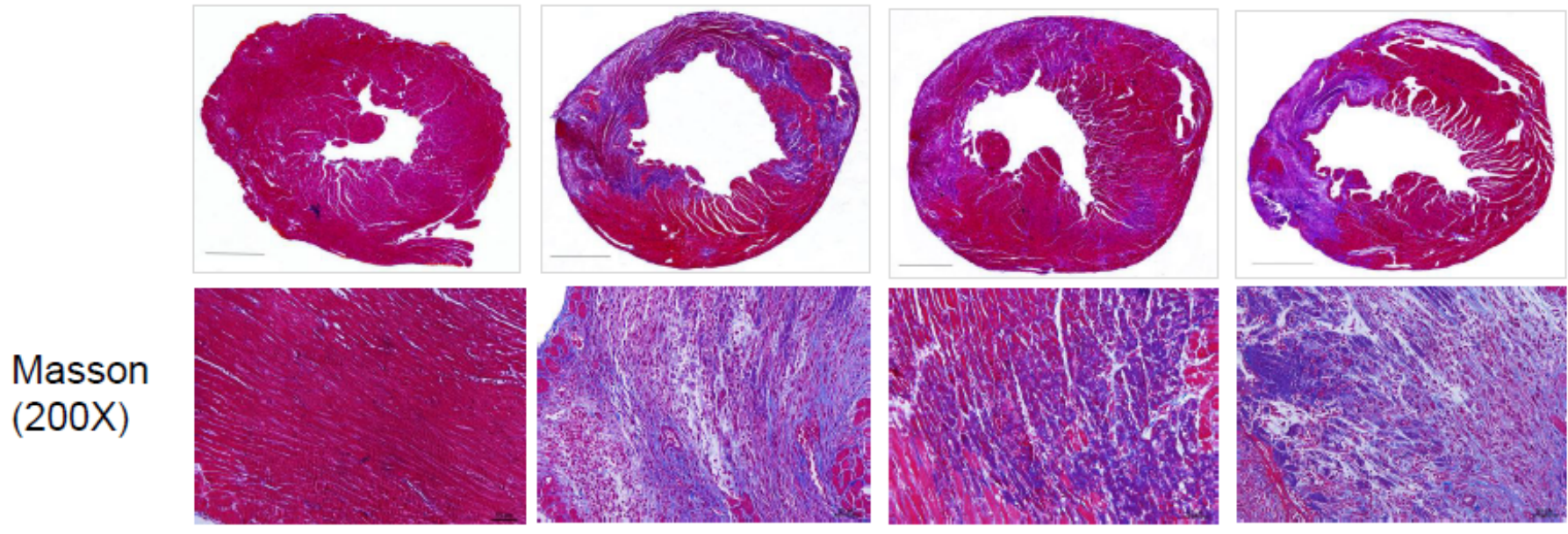

B
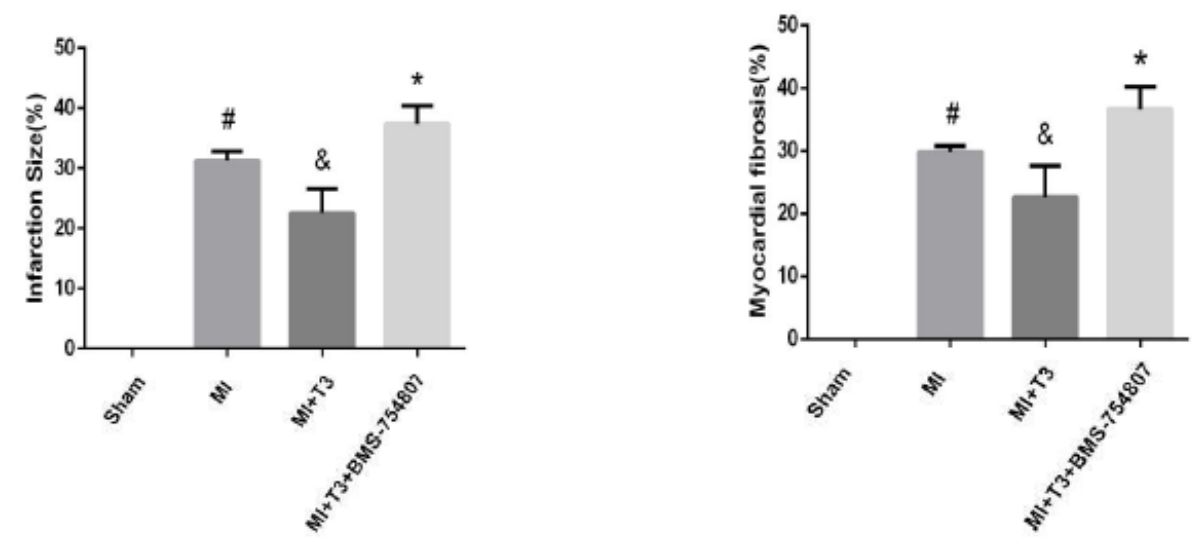

\section{Figure 4}

T3 pretreatment reduces infarct size and ameliorates myocardial fibrosis after MI. (A) Masson's trichrome staining after operation (Scale bar: $2 \mathrm{~mm} ; 200 \times$ magnification, respectively). (B) Infarct size and myocardial fibrosis analysis of mice at 7 th day after operation. Data are expressed as mean \pm SEM $(n=8$ per group). \# $\mathrm{P}<0.05$ versus sham group; \& $\mathrm{P}<0.05$ versus $\mathrm{Ml}$ group, ${ }^{*} \mathrm{P}<0.05$ versus $\mathrm{Ml}+\mathrm{T} 3$ group.
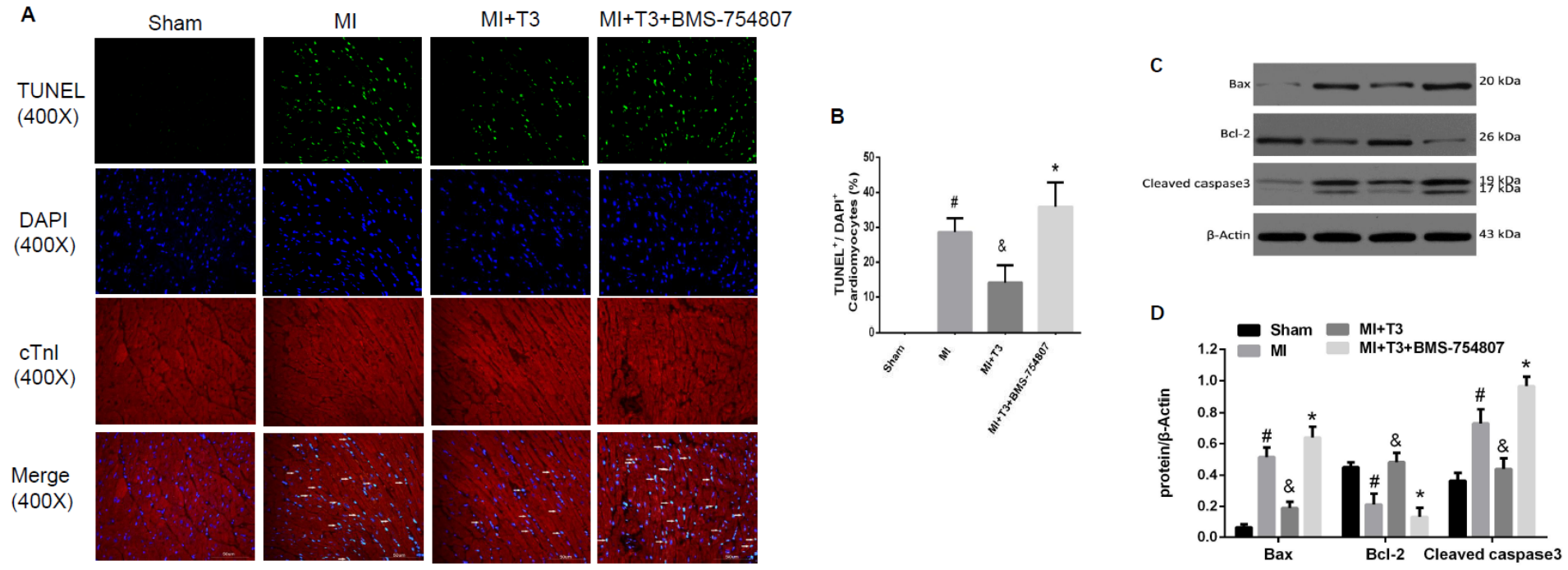


\section{Figure 5}

T3 pretreatment attenuates MI-induced myocardial apoptosis. (A) Images of TUNEL/DAPI/cTnI immunofluorescence staining of hearts within the infarction border zone (400 x magnification). (B) The ratio of TUNEL positive nuclei analysis. (C) Western blots of apoptosis-related protein (Bax, Bcl-2 and cleaved caspase 3) from the infarction border zone of hearts at 7th day after operation. (D) Pooled data from western blots including Bax, Bcl-2 and cleaved caspase 3 protein expression levels. Data are expressed as mean \pm SEM ( $n=8$ per group). \# $P<0.05$ versus sham group; $\& P<0.05$ versus $M I$ group, * $P$ $<0.05$ versus $\mathrm{Ml}+\mathrm{T} 3$ group.

A

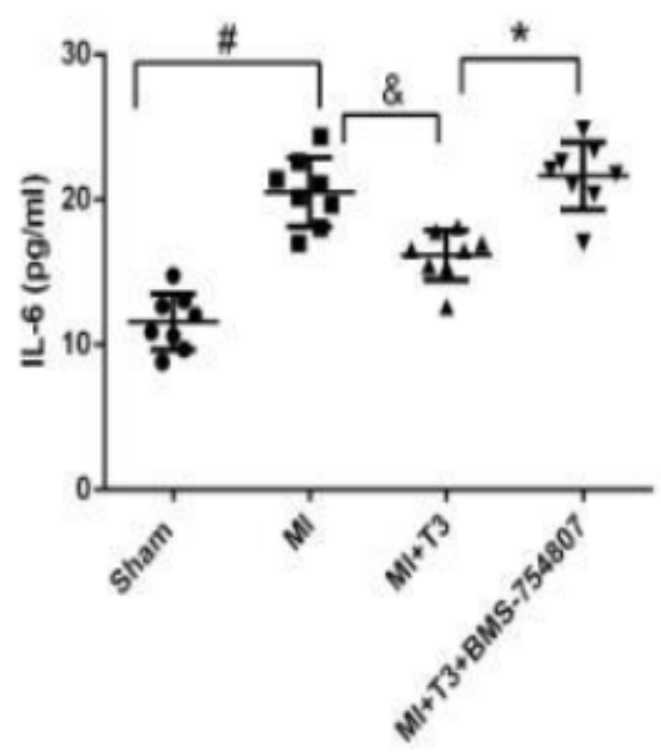

B

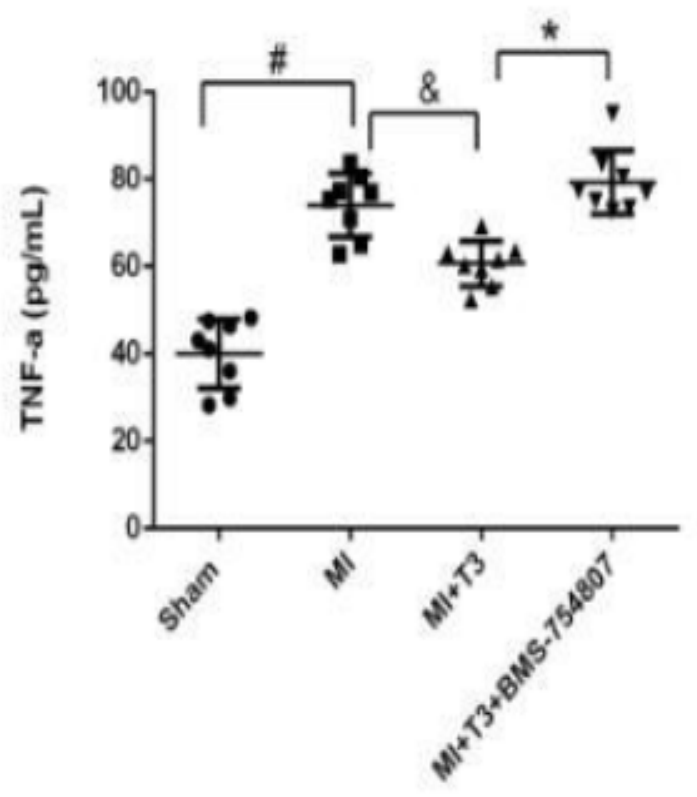

Figure 6

T3 pretreatment inhibits Ml-induced inflammatory responses. (A) Concentration of inflammatory cytokines IL-6 in sera 3 days after operation. (B) Concentration of inflammatory cytokines TNF-a in sera 3 days after operation. IL-6, Interleukin-6; TNF-a, tumor necrosis factor-a. Data are expressed as mean \pm SEM ( $n=8$ per group). \# $P<0.05$ versus sham group; $\& P<0.05$ versus $M I$ group, * $P<0.05$ versus Ml+T3 group. 
A

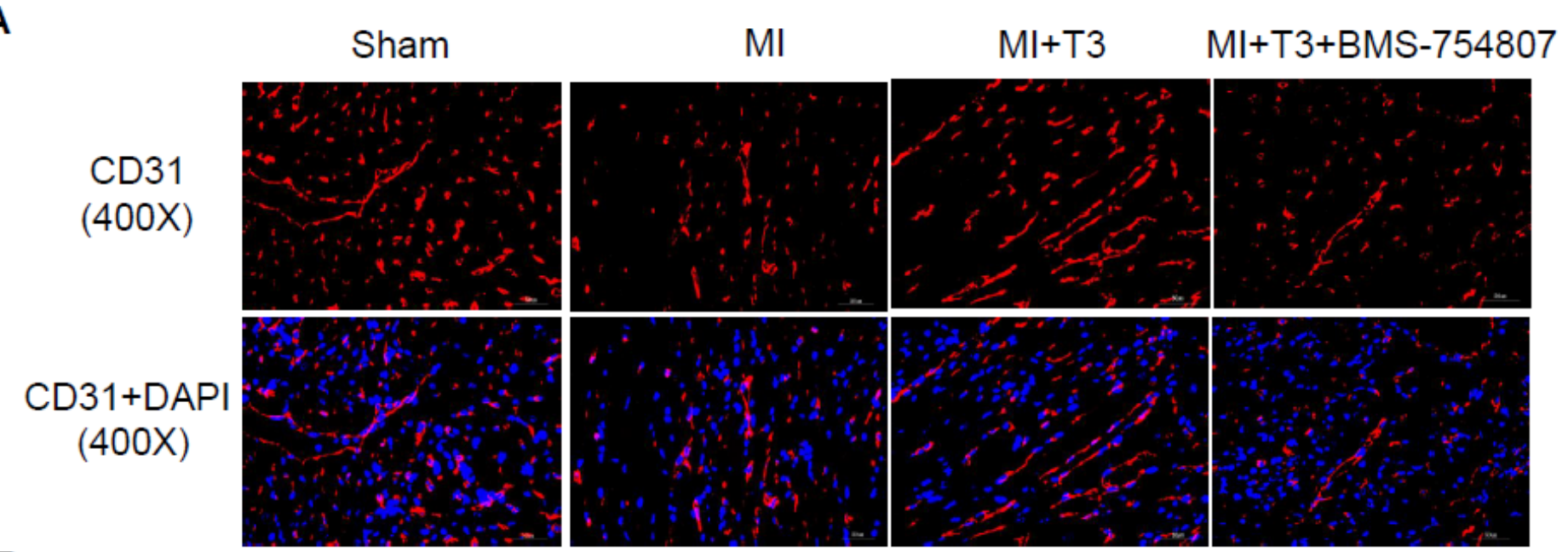

B

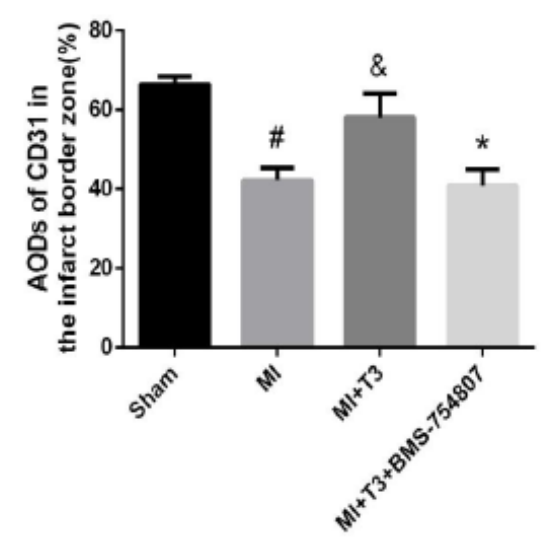

Figure 7

T3 stimulates angiogenesis following MI. (A) Images of CD31 immunofluorescence staining of hearts within the infarction border zone (400 $\times$ magnification). (B) AOD analysis of CD31. Data are expressed as mean \pm SEM ( $n=8$ per group). \# $P<0.05$ versus sham group; $\& P<0.05$ versus $M I$ group, $* P<0.05$ versus $\mathrm{MI}+\mathrm{T} 3$ group. AOD, the average optical densities. 
A
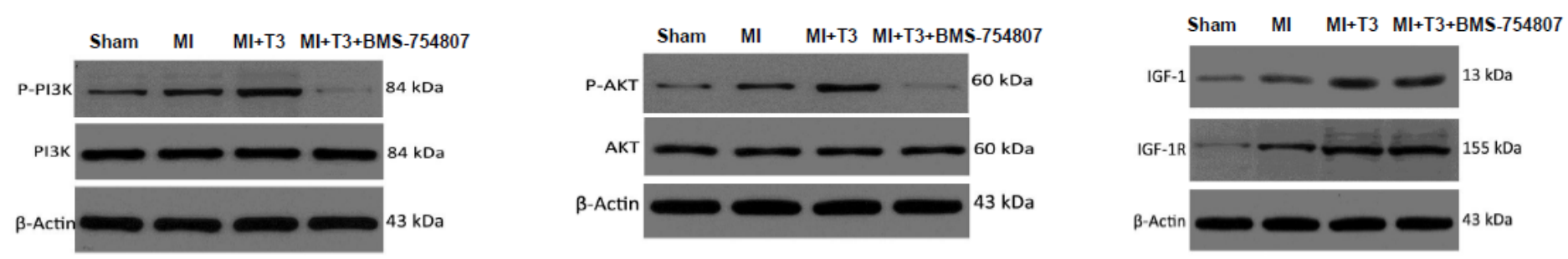

B
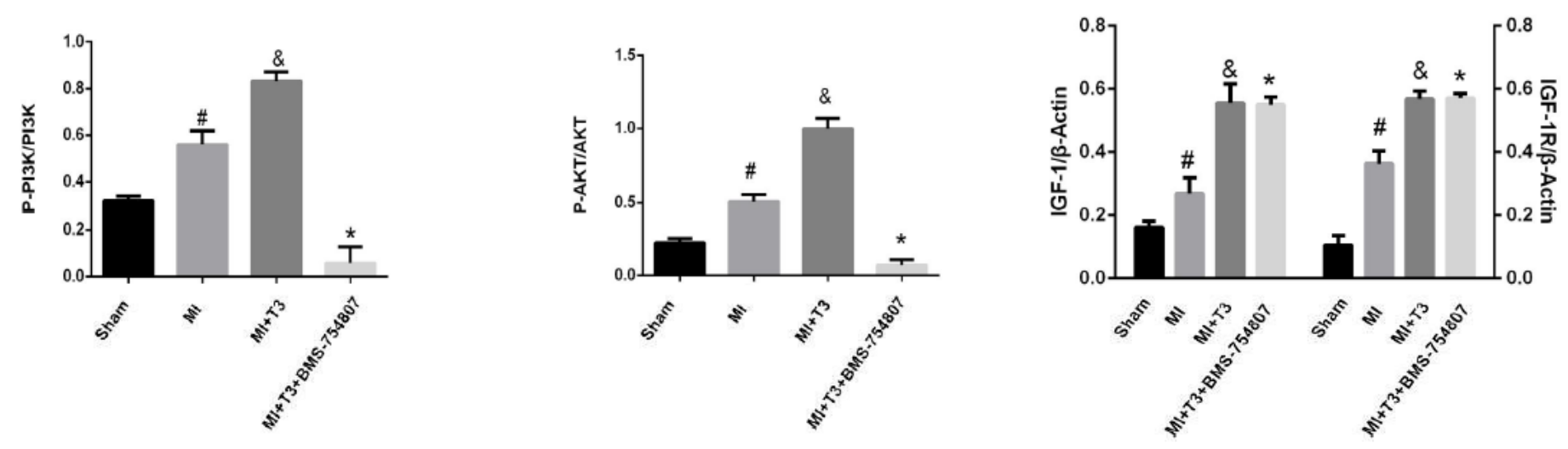

Figure 8

T3 mediates post-MI cardioprotection via the activation of IGF-1/PI3K/AKT signaling pathway. (A) Western blots of IGF-1, IGF-1R, p-PI3K and p-AKT protein from the infarction border zone of hearts at 7th day after operation. (B) Pooled data from western blots including IGF-1, IGF-1R, p-PI3K and p-AKT protein expression levels. Data are expressed as mean \pm SEM ( $n=8$ per group). \# $P<0.05$ versus sham group; \& $P$ $<0.05$ versus $\mathrm{Ml}$ group, ${ }^{*} \mathrm{P}<0.05$ versus $\mathrm{Ml}+\mathrm{T} 3$ group. 